\title{
Collinear Structure Functions of the Nucleon: Status and Future
}

\author{
Sebastian Kuhn*i \\ Old Dominion University, Norfolk, Virginia, USA \\ E-mail: skuhneodu. edu
}

\begin{abstract}
While our ultimate goal is a complete three-dimensional picture of the nucleon in terms of its fundamental constituents, there are still important lessons to be learned about its "onedimensional" collinear parton distribution functions (PDFs) like $f_{1}(x)$ and $g_{1}(x)$. There are rigorous proofs for factorization and universality (process independence) which make these PDFs fundamental. They also appear as limits of Generalized Parton Distributions (GPD) and as integrals of transverse momentum-dependent (TMD) parton distribution functions.
\end{abstract}

Experimentally, the unpolarized structure functions $F_{1}\left(x, Q^{2}\right), F_{2}\left(x, Q^{2}\right)$ have been studied over a huge kinematic range in both variables. Information on the polarized structure functions $g_{1}\left(x, Q^{2}\right), g_{2}\left(x, Q^{2}\right)$ is somewhat more limited, both in kinematics and in statistical precision. In both cases, much less is known about the neutron than the proton, due to the absence of a free neutron target. Accessing these structure functions at large $x$ (where valence quarks dominate) has been challenging due to the high luminosity and the high resolution required. Finally, much information can be extracted from studying higher twist contributions to these structure functions and the connection between the DIS limit and the region where nucleon resonance excitation dominates.

I present an overview of recent experimental results (with special emphasis on the valence region and the transition from quark to hadronic degrees of freedom). I will also give an outlook on the next round of experiments coming online with the energy-upgraded Jefferson Lab electron beam, and future projects like the Electron Ion Collider.

10th Latin American Symposium on Nuclear Physics and Applications

1-6 December, 2013

Montevideo, Uruguay

* Speaker.

${ }^{\dagger}$ This work was supported by the U.S. Department of Energy under contract DE-FG02-96ER40960. 


\section{Introduction}

The partonic structure of protons and neutrons (nucleons) has been studied for 45 years, beginning with the seminal experiments at the Stanford Linear Accelerator Center in the 60's of the previous century $[1,2]$. In particular, through the tool of deep inelastic lepton scattering (which includes electrons, positrons, muons and neutrinos as probes), the (approximate) scaling of the inelastic structure functions $F_{1,2,3}(x)$ was experimentally confirmed and interpreted as representing the momentum distribution of partons (quarks) inside the nucleon, in the form of Parton Distribution Functions (PDFs) [3]. Later, similar experiments on polarized nucleons (and using polarized leptons) studied the spin distribution of these quarks, leading to the surprising conclusion that only a small fraction of the nucleon spin is due to quark helicities [4-6].

Since these beginnings, a massive world-wide effort has led to ever more precise measurements of these structure functions, with an increasing number of probes, and over a huge range of kinematics. On the theory side, the interpretation of these measurements has been put on a firm footing within QCD and they have been used to infer the quark and gluon content of the nucleon, as well as to test perturbative QCD (pQCD) and to extract the running of the strong coupling constant, $\alpha_{s}\left(Q^{2}\right)$. More recently, a richer and more detailed picture of the nucleon in terms of truly 3-dimensional parton distributions (so-called Generalized Parton Distributions - "GPDs" and Transverse Momentum Dependent PDFs - "TMDs") has emerged. Ultimately, our goal is to extract both the longitudinal (collinear) and transverse motion and spin of all constituents (quarks and gluons) of the nucleon and to relate them to its gross features - its mass, spin, magnetic moment, charge distribution etc.. In this quest, collinear PDFs are still playing a fundamental role, with a large experimental program underway at existing facilities (COMPASS at CERN and RHIC at BNL) and planned for the energy-upgraded Jefferson Lab at $12 \mathrm{GeV}$ and a future Electron-Ion Collider. There are two main reasons for this continued importance:

1. There is a rigorous proof that collinear structure functions factorize into two parts: the fundamental quark scattering cross section on the hard (high momentum) scale, which can be calculated precisely in pQCD, and the PDFs on the soft (low momentum) scale [7]. The latter have been proven to be "universal" (independent of the reaction under study) and can therefore be rightfully interpreted as encoding the internal structure of the nucleon.

2. Collinear structure functions are the limiting cases for GPDs (for momentum transfer $t \rightarrow$ 0 ) and integrals of the TMDs (integrated over all transverse momenta). Since they can be measured most precisely, they provide important constraints on models of GPDs and TMDs, which are needed to interpret the experimental data (which only indirectly measure these quantitites).

In the following, I will give a brief overview over the interpretation of PDFs (Section 2) and the experimental determination of structure functions (Section 3) from which they can be extracted. I then highlight a few recent experimental results on unpolarized (Section 4) and polarized (Section 5) structure functions. Finally, I summarize the main points and give an outlook on future experimental programs (Section 6). 


\section{Partonic Structure of the Nucleon}

For a basic understanding of the significance of parton distribution functions, it helps to consider the following simplified picture (also known as "naïve parton model"):

- Assume a hadron (e.g., a proton) moving along the z-direction at very high momentum (the "infinite momentum frame").

- Its four-momentum $P^{\mu}$ can be expressed in terms of its light cone momentum $P^{+}=P^{0}+P^{3}=$ $E+P_{z} \gg M$ (together with the smaller components, $P^{-}$and $\vec{P}_{T}$ ).

- Accordingly, we can define the light cone momentum of any of its constituents (quarks, antiquarks, and gluons), $p^{+}=p^{0}+p^{3}$, or the momentum fraction $x=p^{+} / P^{+}$. The advantage of the latter quantity is that it is invariant under boosts along the $\mathrm{z}$ axis.

- We call $f_{1}^{i}(x)$ the probability of finding a parton of type $i$ with momentum fraction $x$ inside the nucleon. (Another common notation uses $q_{i}(x)$ for quarks and $G(x)$ for gluons; $i=u, d, s \ldots$ ).

- If we use an electromagnetic probe, we have to sum over all charged partons (i.e., quarks), weighted by their (squared) charges $z_{i}$. The result is the structure function $F_{1}(x)=\frac{1}{2} \sum_{i} z_{i}^{2} f_{1}^{i}(x)$.

For a concrete example, let us consider inclusive lepton scattering where a lepton with initial energy $E$ and momentum $\vec{k}$ (four-momentum $k^{\mu}$ ) scatters off the hadron to a final energy of $E^{\prime}$ and momentum $\vec{k}^{\prime}$ (four-momentum $k^{\prime \mu}$ ). We can define the four-momentum transfer, $q^{\mu}=k^{\mu}-k^{\prime \mu}$, and its square, $Q^{2}=-q^{\mu} q_{\mu}$. Similarly, we define the energy transfer $v=E-E^{\prime}$. In the one photon exchange (Born) approximation, the lepton interacts with the target through the exchange of a virtual photon with energy $v$ and momentum $\vec{q}=\vec{k}-\vec{k}^{\prime}$. If we choose our coordinate system such that the virtual photon travels in the negative z-direction $(\vec{q}=(0,0,-q))$, we can define the light cone momentum of that photon as $v-q$. In the Bjorken limit of large $Q^{2}$ and $v$, this virtual photon is absorbed by a single quark that can be treated as "asymptotically free" (a fundamental feature of QCD). Therefore, after the absorption, this quark has light cone momentum $p^{+}+v-q$. It will now travel in the negative z-direction, and therefore, this light cone momentum should be equal to its energy minus its final state momentum. In the limit where we can ignore quark masses (a few MeV for $u$ and $d$ quarks) and transverse momenta, momentum and energy have the same magnitudes, and therefore this light cone momentum must be zero. Hence, we require $p^{+} \approx q-v$ and $x \approx \xi=(q-v) / P^{+}$. Since this variable is boost-invariant, we can evaluate it in the rest frame of the initial nucleon: $\xi_{L a b}=\left(q_{L a b}-v_{L a b}\right) / M$. (The variable $\xi$ was originally introduced by $\mathrm{O}$. Nachtmann [8].) At very large $Q^{2} \gg M^{2}$, this variable becomes identical to the Bjorken scaling variable $x_{B j}=Q^{2} / 2 P^{\mu} q_{\mu}$ which simplifies to $Q^{2} / 2 M v_{L a b}$ in the target rest frame. Therefore, in the Bjorken limit the probability for the lepton scattering process described above will be proportional to the structure function $F_{1}(x)$ defined above, with $x \approx \xi \approx x_{B j}$. In practice, the Bjorken variable is most widely used, which means that corrections of order $Q^{2} / M^{2}$ (target mass corrections) have to be applied. (There are other corrections to this simple picture which I discuss in the next section).

The picture painted above becomes a bit more complicated once spin degrees of freedom are incorporated. In the same high momentum frame as before, one can describe the hadron (nucleon) spin through its projection along the z-direction, the helicity $H$, as well as its transverse component 
$\vec{S}_{T}$. (Note that these two components have to be treated separately for a highly relativistic system as considered here, because rotations and Lorentz boosts do not commute. Also, it is clear that one cannot measure both components simultaneously as they don't commute with each other). Similarly, we introduce the parton helicity $h$ and transverse spin $\vec{s}_{T}$. Of all possible combinations of these new quantities with each other and the unit vector $\hat{z}$, only two are invariant under rotation and under parity: $h H$ and $\vec{s}_{T} \cdot \vec{S}_{T}$. This allows us to introduce two more parton distributions:

$$
\begin{array}{r}
g_{1}^{i}(x)=<h H>f_{1}^{i}(x) \\
h_{1}^{i}(x)=<\vec{s}_{T} \cdot \vec{S}_{T}>f_{1}^{i}(x) .
\end{array}
$$

The first one is often written as $g_{1}^{i}(x)=\Delta q_{i}(x)=q_{i}^{\Uparrow \uparrow}-q_{i}^{\Uparrow \downarrow}$ for quarks and can be interpreted as the probability of finding a quark with light cone momentum fraction $x$ and with its helicity aligned with the nucleon helicity, minus the same probability for anti-aligned quark helicity. The equivalent expression for gluons is $\Delta G(x)$. In complete analogy to the "unpolarized" structure function $F_{1}$, we can then write $g_{1}(x)=\frac{1}{2} \sum_{i} z_{i}^{2} g_{1}^{i}(x)=\frac{1}{2} \sum_{i} z_{i}^{2} \Delta q_{i}(x)$ for the spin structure function $g_{1}$. One can access $g_{1}$ in inclusive lepton scattering, as well, by polarizing the target along the z-direction and measuring the cross section difference for leptons with positive and negative helicity. The electron helicity is partially transferred to the spin component of the virtual photon along the z-axis. In turn, the virtual photon can only be absorbed by a quark with its own helicity opposite to that of the photon, since the photon has spin 1 and the final state quark must of course have spin 1/2.

The second distribution in Eq. 2.2 is called transversity; it is on equal footing with the other two, but not accessible in inclusive scattering. Instead, it can be accessed through Drell-Yan processes or, indirectly, through semi-inclusive DIS (SIDIS). SIDIS can also be used to "tag" the flavor of the struck quark, which is likely to be contained in the fastest (highest energy) hadron produced in the final state. Further distribution functions can be defined if one also considers the transverse momentum $p_{T}$ of the parton; these are the Transverse Momentum Dependent PDFs ("TMD"). They are not collinear and are not considered further in the following.

\section{Structure Functions}

In the previous section, we have outlined how one can in principle measure the structure functions $g_{1}$ and $F_{1}$ and how they can be interpreted in terms of parton distributions in the nucleon. In practice, several complications arise in both steps.

First, there are additional structure functions that are needed to fully describe inclusive lepton scattering. These additions come about because virtual photons (unlike real ones) can have electric field components along their direction of motion $(\hat{q})$. The fractional value of this "longitudinal polarization" (not to be confused with spin polarization) is expressed by the parameter

$$
\varepsilon=\left(1+2\left[1+1 / \gamma^{2}\right] \tan ^{2} \frac{\theta_{e}}{2}\right)^{-1}
$$

in the target rest frame $\left(\theta_{e}\right.$ is the electron scattering angle, and $\left.\gamma=\sqrt{Q^{2}} / v\right)$. One can parametrize the contribution of these "longitudinal photons" through the ratio $R=\sigma_{L} / \sigma_{T}$ of longitudinal over 
transverse virtual photon absorption cross sections, and the virtual photon transverse-longitudinal interference asymmetry $A_{2} . R$ goes to zero in the Bjorken limit, and $A_{2}$ is constrained by the Soffer inequality [9]), $\left|A_{2}\right| \leq \sqrt{R\left(1+A_{1}\right) / 2}$.

For completeness, we also express other often-used structure functions in terms of this set:

$$
\begin{aligned}
& F_{2}\left(x, Q^{2}\right)=2 x F_{1}\left(x, Q^{2}\right) \frac{1+R\left(x, Q^{2}\right)}{1+\gamma^{2}} \rightarrow 2 x F_{1}(x) \\
& g_{2}\left(x, Q^{2}\right)=g_{T}\left(x, Q^{2}\right)-g_{1}\left(x, Q^{2}\right) ; g_{T}\left(x, Q^{2}\right)=F_{1}\left(x, Q^{2}\right) \frac{A_{2}\left(x, Q^{2}\right)}{\gamma} \rightarrow \int_{x}^{1} g_{1}(y) \frac{d y}{y} \\
& A_{1}\left(x, Q^{2}\right)=\frac{g_{1}\left(x, Q^{2}\right)-\gamma^{2} g_{2}\left(x, Q^{2}\right)}{F_{1}\left(x, Q^{2}\right)}=\left(1+\gamma^{2}\right) \frac{g_{1}\left(x, Q^{2}\right)}{F_{1}\left(x, Q^{2}\right)}-\gamma A_{2}\left(x, Q^{2}\right) \rightarrow \frac{g_{1}(x)}{F_{1}(x)}
\end{aligned}
$$

The right-most expressions in Eq. 3.2 show the asymptotic behavior in the Bjorken limit and at leading twist (see below). The expression for $g_{T}$ is the Wandzura-Wilczek relation [10].

For an electron beam with helicity $+(-)$ scattering off a nucleon with its spin oriented opposite to the electron beam direction, one can write the cross section as

$$
\frac{d \sigma^{+(-)}}{d \Omega d E^{\prime}}=\sigma_{M}\left[\frac{F_{2}}{v} \frac{1+\varepsilon R}{\varepsilon(1+R)} \pm 2 \tan ^{2} \frac{\theta_{e}}{2}\left(\frac{E+E^{\prime} \cos \theta_{e}+Q^{2} / v}{M v} g_{1}-\frac{2 x F_{1} A_{2}}{\sqrt{Q^{2}}}\right)\right]
$$

where the Mott cross section

$$
\sigma_{M}=\frac{4 E^{\prime 2} \alpha^{2} \cos ^{2} \frac{\theta}{2}}{Q^{4}}
$$

A similar expression exists for the case where the nucleon spin is transverse to the beam direction. Combining both measurements allows one to extract both $g_{1}$ (or $A_{1}$ ) and $A_{2}$ (or $g_{2}$ ) separately.

From the theory side, the naïve parton model has to be replaced by a rigorous perturbative approach (pQCD) which takes into account QCD radiative corrections and the mixing of gluon and quark contributions through the DGLAP evolution equations [11-13] for $g_{1}$ and $f_{1}$. As a consequence, both of theses structure functions become (logarithmically) dependent on $Q^{2}$ as well as on $x$, which allows us to gain (indirect) information on gluon PDFs even from inclusive lepton scattering. For lower values of $Q^{2} \approx M^{2}$, both target mass correction (see above) and higher twist contributions $[14,15]$ become important (they modify the structure functions with additional terms proportional to powers of $1 / Q$ ). The latter parametrize the deviations from the "asymptotically free parton" picture due to quark-quark and quark-gluon correlations; far from being a mere nuisance, they open access to additional information about the internal structure of the nucleon. At moderate $Q^{2}$ and large $x$, the invariant mass of the final state $W=\sqrt{M^{2}+(1 / x-1) Q^{2}}$ becomes comparable to that of resonant excitations of the nucleon $(W \leq 2 \mathrm{GeV})$ which modify the structure functions significantly. Somewhat surprisingly, when averaged over those "resonance bumps", structure functions seem to still follow the general trend from the Bjorken region; this phenomenon of "quark-hadron duality" [16] has generated a lot of interest as a means to unify the partonic picture of the nucleon with hadron degrees of freedom that govern its low-energy properties. Finally, in the limit $Q^{2} \rightarrow 0$ structure functions and their integrals can be used to test low-energy theorems and effective theories like Chiral Perturbation Theory $(\chi \mathrm{PT})[17-19]$ in a regime where pQCD is clearly not applicable. In the following, we will discuss a few recent experiments aimed at all of these different kinematic regimes. 


\section{Unpolarized Structure Functions - Recent Results}

The "unpolarized" structure functions $F_{1}, F_{2}$ and $R$ (as well as the related neutrino scattering structure function $F_{3}$ ) have been measured over a huge kinematic range at a large number of facilities - from the (now decommissioned) high-energy facilities like the Tevatron at Fermilab (Illinois/USA) and HERA at DESY (Hamburg/Germany) to the low-energy experiments at Jefferson Lab (Virginia/USA). A rather complete summary of all the world data on inclusive lepton scattering can be found in a paper by the HERMES collaboration [20] and the compilation by the Particle Data Group [21]. New results are coming from the highest energies available at the Large Hadron Collider and soon from the energy-upgraded Jefferson Lab at $11 \mathrm{GeV}$. Several groups continue to fit these data in the framework of the DGLAP evolution equations [11 - 13] to extract parton densities for each individual quark flavor and for gluons - see for instance the results in [22].

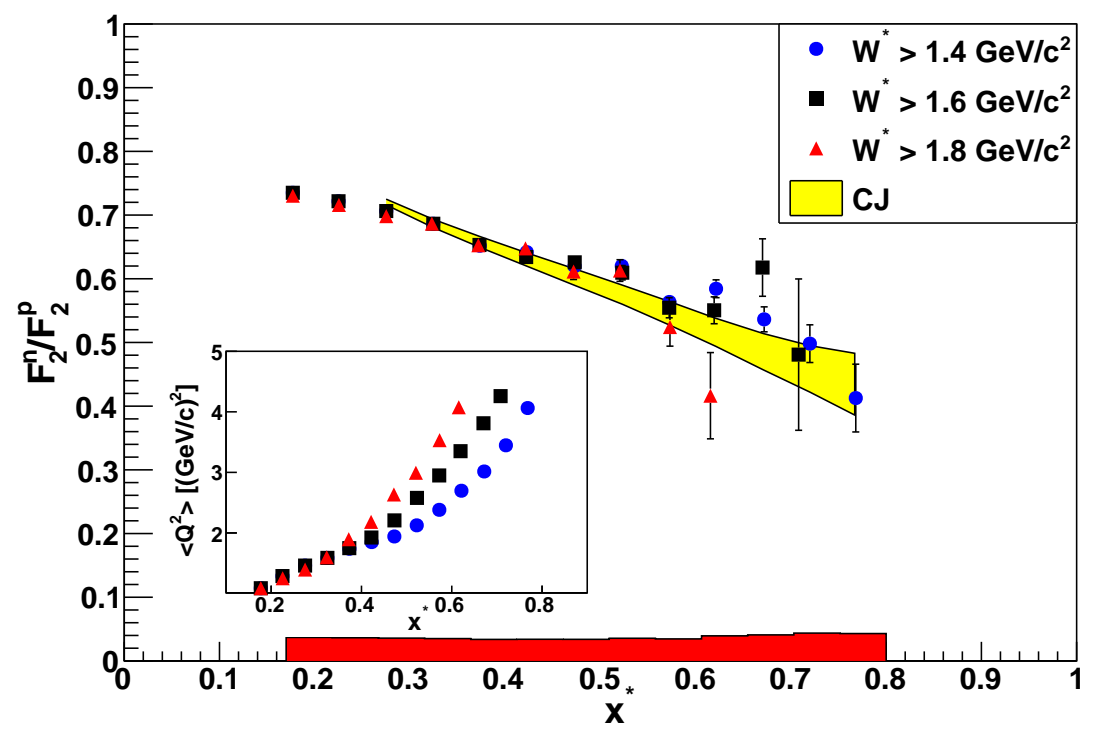

Figure 1: (Color online) Results for the ratio of the neutron to proton structure functions $F_{2}^{n} / F_{2}^{p}(x)$ (integrated over $Q^{2}>1 \mathrm{GeV}^{2} / c^{2}$ and three different minimum values for $W$ ) from the BONuS experiment. The present uncertainty range from the CJ fit [23] is shown by the yellow shaded band. Systematic uncertainties are shown as the red shaded band at the bottom. The data are cross-normalized to the average of the CJ fit at $x=0.32$. The inset shows the average $Q^{2}$ for each data point, separately for the three lower $W$ limits.

A topic of particular interest is the behavior of the PDFs at very large $x$ (close to 1) where valence quarks are expected to dominate (i.e., the two up and one down quark that carry the quantum numbers of the proton). On the one hand, both phenomenological models like the constituent quark model and pQCD calculations make specific predictions for quantities like the ratio of down over up quark distributions $d(x) / u(x)$ as $x \rightarrow 1$ that should be tested. At the same time, quark distributions at high $x$ and moderate $Q^{2}$ are related to lower- $x$ distributions at the very high $Q^{2}$ relevant for the Large Hadron Collider, through pQCD evolution. A recent pQCD analysis [23] explored this kinematic region by relaxing standard cuts on final state mass $W$ to include high- $x$ results from fixed target experiments like the ones at Jefferson Lab. To extract both $u(x)$ and $d(x)$, one needs 
to include data on the neutron in these fits. However, since free neutron targets are not available, one has to use measurements on deuterium or other nuclei, which require models of nuclear effects like binding and Fermi motion to extract neutron information, with correspondingly large model uncertainties.

One way to avoid these model uncertainties is offered by a technique called "spectator tagging". Instead of just measuring the inclusive scattering cross section on deuterium, ${ }^{2} \mathrm{H}\left(e, e^{\prime}\right)$, one detects a proton in coincidence with the scattered electron, ${ }^{2} \mathrm{H}\left(e, e^{\prime} p_{s}\right)$. By selecting protons that move with low momentum $(<100 \mathrm{MeV} / c)$ and backwards relative to the momentum transfer direction $\hat{q}\left(\theta_{p q}>100^{\circ}\right)$, one can ensure that they are mere "spectators" (hence the index " $p_{s}$ ") to a scattering process occurring on the neutron in deuterium. Furthermore, by momentum conservation one can "tag" the initial motion of the struck neutron, thereby eliminating the smearing effect of Fermi motion. Selecting low momenta minimizes both final state interaction effects and guarantees that the struck neutron is close to a free one, with energy not much less than $E=\sqrt{M^{2}+\vec{p}^{2}}$. A pioneering experiment along these lines was recently conducted at Jefferson Lab by the "BONuS" collaboration [24-26]. The results for the ratio of neutron to proton structure functions, from which $d(x) / u(x)$ can be extracted, is shown in Fig. 1. Future extensions of this approach to higher energies will allow us to definitely pin down the asymptotic behavior of $d(x) / u(x)$ as $x \rightarrow 1$.

\section{Polarized Structure Functions - Recent Results}

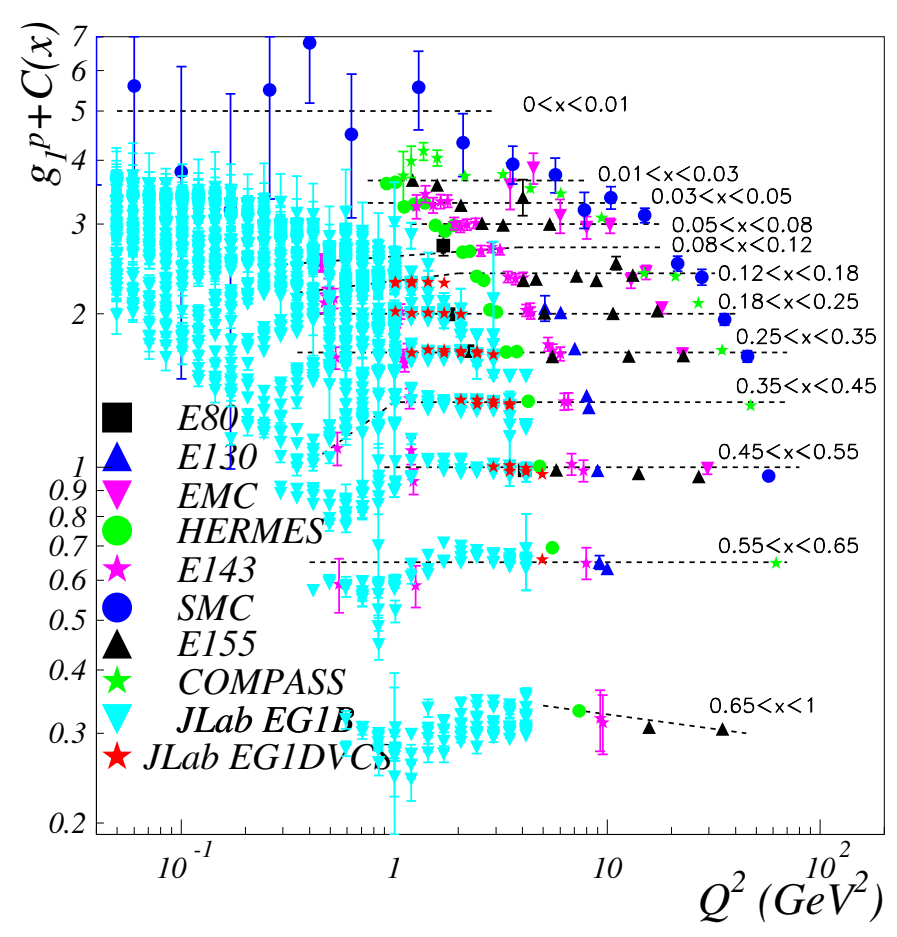

Figure 2: Experimental data for the spin structure function $g_{1}^{p}$ of the proton, covering both the DIS and the low $Q^{2}$, low $W$ region. 
Spin dependent PDFs have also been studied at several experimental facilities (SLAC, CERN, DESY, Jefferson Lab and RHIC); however, the data cover a smaller kinematic region than the unpolarized ones. Figure 2 gives an overview of the world data on the spin structure function $g_{1}^{p}$ of the proton; similar data exist for the deuteron and for polarized ${ }^{3} \mathrm{He}$, which can be considered as an effective polarized neutron target (albeit with nuclear binding effects to be corrected for, as in the case of unpolarized structure functions). The limited range in $Q^{2}$ is a consequence of the fact that no polarized lepton-nucleon colliders have been operated so far; this is one of the major driving forces behind the proposal for a new electron-ion collider (EIC). (Note that many of the very copious data from Jefferson Lab are in or near the resonance region, where pQCD evolution does not apply or is at least complicated by higher twist effects). At present, only very limited information on the helicity-dependent gluon PDFs can be extracted from DIS data; this is augmented with measurements of semi-inclusive final states (high transverse momentum or charmed hadron production) and proton-proton collisions at RHIC. The latter experiments are also beginning to constrain sea quark helicity distributions, through $W$ boson production in pp collisions [27].

Again, several groups are actively analyzing all of these data to extract the best possible information on helicity-dependent quark and gluon distributions, see for example [28, 29]. The latter of these two groups (the "JAM" collaboration at Jefferson Lab) once again focuses on the moderate$Q^{2}$, high- $x$ region sensitive to valence quarks by carefully applying corrections for higher twists, target mass and nuclear binding.

At even lower $Q^{2}$, spin structure function measurements can determine higher twist matrix elements, and can test quark-hadron duality as well as effective theories like chiral perturbation theory (see Section 3). Of particular interest are moments of $g_{1}$ and $g_{2}$, for which several sum rules exist (from the real photon point - the famous Gerasimov-Drell-Hearn sum rule [30, 31] - to the DIS limit - the equally famous Bjorken sum rule $[32,33]$ ). Many of the Jefferson Lab data were specifically measured towards this goal. A summary of the status of spin structure functions of about 5 years ago can be found in [34]. Since then, new data have been collected by the SANE collaboration in Jefferson Lab's Hall C ( $g_{1}$ and especially $g_{2}$ in the valence region), by the Hall A collaboration ( $g_{2}$ for protons at small $Q^{2}$ and for ${ }^{3} \mathrm{He}$ ), and first results have become available from the EG4 and EG1-DVCS collaborations in Hall B ( $g_{1}$ for proton and deuteron over a huge kinematic range, $\left.0.02(\mathrm{GeV} / c)^{2}<Q^{2}<5(\mathrm{GeV} / c)^{2}\right)$.

\section{Outlook and Summary}

From the few examples given in these pages, it is hopefully apparent how rich both the theoretical landscape and the experimental efforts in the area of collinear structure functions continue to be. Many already collected data sets (from Jefferson Lab, COMPASS, HERMES, RHIC and other places) are still being analyzed, and new data are being taken. Looking forward, two major new thrusts will continue to expand our knowledge of collinear PDFs:

- Jefferson Lab has just completed the $12 \mathrm{GeV}$ upgrade of its accelerator, and is poised to begin a decades-long program of new measurements in four Halls. Many of the already approved experiments in this program address the valence structure of the nucleon, through measurements of both polarized and unpolarized structure functions (including those on the neutron) 
to the highest possible $x \approx 0.8$, with unprecedented precision and $Q^{2}$ coverage. These data will finally pin down the asymptotic behavior as $x \rightarrow 1$ and clarify the contributions of parton spin and orbital angular momentum to the proton spin.

- Beyond this program, the next step necessary to complete our picture of the internal landscape of the nucleon is the construction and operation of an electron-ion collider (EIC), with polarized light ions like proton, deuteron and ${ }^{3} \mathrm{He}$ at energies of $100 \mathrm{GeV}$ or more. Such a collider would vastly expand the $Q^{2}$ coverage, enabling a similar precision in DGLAP analyses of spin structure functions as were available through HERA. It would also finally extend these measurements to very low $x \approx 10^{-4}$, where gluon degrees of freedom are important and significant surprises (as in the case of HERA) may await us. Such an EIC is emerging as the next flagship facility for the international nuclear and hadronic physics community.

\section{References}

[1] E. D. Bloom et al., High-Energy Inelastic e p Scattering at 6-Degrees and 10-Degrees, Phys. Rev. Lett. 23 (1969) 930-934.

[2] M. Breidenbach, J. I. Friedman, H. W. Kendall, E. D. Bloom, D. Coward, et al., Observed Behavior of Highly Inelastic electron-Proton Scattering, Phys.Rev.Lett. 23 (1969) 935-939.

[3] R. P. Feynman, Very high-energy collisions of hadrons, Phys. Rev. Lett. 23 (Dec, 1969) 1415-1417.

[4] M. J. Alguard et al., Deep inelastic scattering of polarized electrons by polarized protons, Phys. Rev. Lett. 37 (1976) 1261.

[5] G. Baum et al., A new measurement of deep inelastic e p asymmetries, Phys. Rev. Lett. 51 (1983) 1135.

[6] EMC Collaboration, J. Ashman et al., An investigation of the spin structure of the proton in deep inelastic scattering of polarized muons on polarized protons, Nucl. Phys. B328 (1989) 1.

[7] J. C. Collins, D. E. Soper, and G. F. Sterman, Factorization of Hard Processes in QCD, Adv.Ser.Direct.High Energy Phys. 5 (1988) 1-91, [hep-ph/ 0409313$].$

[8] O. Nachtmann, Inequalities for structure functions of deep inelastic lepton-nucleon scattering giving tests of basic algebraic structures, Nucl. Phys. B 38 (1972) 397.

[9] J. Soffer and O. Teryaev, Improved positivity bound for deep inelastic scattering on transversely polarized nucleon, hep-ph/9906455.

[10] S. Wandzura and F. Wilczek, Sum Rules for Spin Dependent Electroproduction: Test of Relativistic Constituent Quarks, Phys.Lett. B72 (1977) 195.

[11] Y. L. Dokshitzer, Calculation of the Structure Functions for Deep Inelastic Scattering and e+eAnnihilation by Perturbation Theory in Quantum Chromodynamics, Sov. Phys. JETP 46 (1977) 641-653.

[12] V. N. Gribov and L. N. Lipatov, Deep inelastic e p scattering in perturbation theory, Sov. J. Nucl. Phys. 15 (1972) 438-450.

[13] G. Altarelli and G. Parisi, Asymptotic Freedom in Parton Language, Nucl. Phys. B126 (1977) 298.

[14] E. V. Shuryak and A. I. Vainshtein, Theory of power corrections to deep inelastic scattering in quantum chromodynamics. 1. q**2 effects, Nucl. Phys. B199 (1982) 451. 
[15] E. V. Shuryak and A. I. Vainshtein, Theory of power corrections to deep inelastic scattering in quantum chromodynamics. 2. $q^{* * 4}$ effects: Polarized target, Nucl. Phys. B201 (1982) 141.

[16] E. D. Bloom and F. J. Gilman, Scaling and the behavior of nucleon resonances in inelastic electron-nucleon scattering, Phys. Rev. D4 (1971) 2901.

[17] X. Ji and J. Osborne, Generalized sum rules for spin dependent structure functions of the nucleon, J. Phys. G 27 (2001) 127.

[18] V. Bernard, T. R. Hemmert, and U.-G. Meissner, Spin structure of the nucleon at low energies, Phys. Rev. D67 (2003) 076008, [hep-ph/ 0212033$].$

[19] C. W. Kao, T. Spitzenberg, and M. Vanderhaeghen, Burkhardt-cottingham sum rule and forward spin polarizabilities in heavy baryon chiral perturbation theory, Phys. Rev. D67 (2003) 016001 , [hep-ph/0209241].

[20] HERMES Collaboration, A. Airapetian et al., Inclusive Measurements of Inelastic Electron and Positron Scattering from Unpolarized Hydrogen and Deuterium Targets, JHEP 1105 (2011) 126, [1103.5704].

[21] J. Beringer et al., Review of Particle Physics (Particle Data Group), Phys. Rev. D 86 (2012) 010001.

[22] S. Alekhin, J. Bluemlein, and S. Moch, The ABM parton distributions tuned to LHC data, 1310.3059 .

[23] J. F. Owens, A. Accardi, and W. Melnitchouk, Global parton distributions with nuclear and finite- $Q^{2}$ corrections, Phys. Rev. D 87 (2013) 094012, [1212.1702].

[24] H. C. Fenker, V. Burkert, R. Ent, N. Baillie, J. Evans, et al., BoNuS: Development and Use of a Radial TPC using Cylindrical GEMs, Nucl.Instrum.Meth. A592 (2008) 273-286.

[25] CLAS Collaboration, N. Baillie et al., Measurement of the neutron F2 structure function via spectator tagging with CLAS, Phys.Rev.Lett. 108 (2012) 199902, [1110.2770].

[26] CLAS Collaboration, S. Tkachenko et al., Measurement of the nearly free neutron structure function using spectator tagging in inelastic ${ }^{2} H(e, e$ 'p)X scattering with CLAS, 1402.2477.

[27] Collaboration STAR Collaboration, J. R. Stevens, The STAR W Program at RHIC, 1302.6639.

[28] D. de Florian, R. Sassot, M. Stratmann, and W. Vogelsang, QCD Spin Physics: Partonic Spin Structure of the Nucleon, Prog.Part.Nucl.Phys. 67 (2012) 251-259, [1112. 0904 ].

[29] P. Jimenez-Delgado, W. Melnitchouk, and J. Owens, Parton momentum and helicity distributions in the nucleon, J.Phys. G40 (2013) 093102, [1306.6515].

[30] S. B. Gerasimov, A sum rule for magnetic moments and the damping of the nucleon magnetic moment in nuclei, Sov. J. Nucl. Phys. 2 (1966) 430-433.

[31] S. D. Drell and A. C. Hearn, Exact sum rule for nucleon magnetic moments, Phys. Rev. Lett. 16 (1966) 908-911.

[32] J. D. Bjorken, Applications of the chiral u(6) x (6) algebra of current densities, Phys. Rev. 148 (1966) 1467-1478.

[33] J. D. Bjorken, Inelastic scattering of polarized leptons from polarized nucleons, Phys. Rev. D1 (1970) 1376-1379.

[34] S. E. Kuhn, J. P. Chen, and E. Leader, Spin Structure of the Nucleon - Status and Recent Results, Prog. Part. Nucl. Phys. 63 (2009) 1-50, [hep-ph/0812.3535]. 